\title{
Ultrasound for Critical Care Physicians: Now My Heart Is Even More Full
}

\author{
Bilal Jalil, MD \\ Michel Boivin, MD
}

Division of Pulmonary, Critical Care and Sleep Medicine

University of New Mexico School of Medicine

Albuquerque, NM

A 49-year-old man with type 2 diabetes, intravenous drug abuse and heart failure presented to the emergency room with 2 weeks of progressively worsening chest pain, lower extremity swelling and shortness of breath. The patient was found to have an elevated troponin as well as brain natriuretic peptide and the absence of ischemic electrocardiogram findings. The patient was admitted to the medical ICU for hypoxic respiratory failure and shock of uncertain etiology. Clinically he seemed to be in decompensated heart failure and a bedside echocardiogram was performed (Figures 1 and 2).

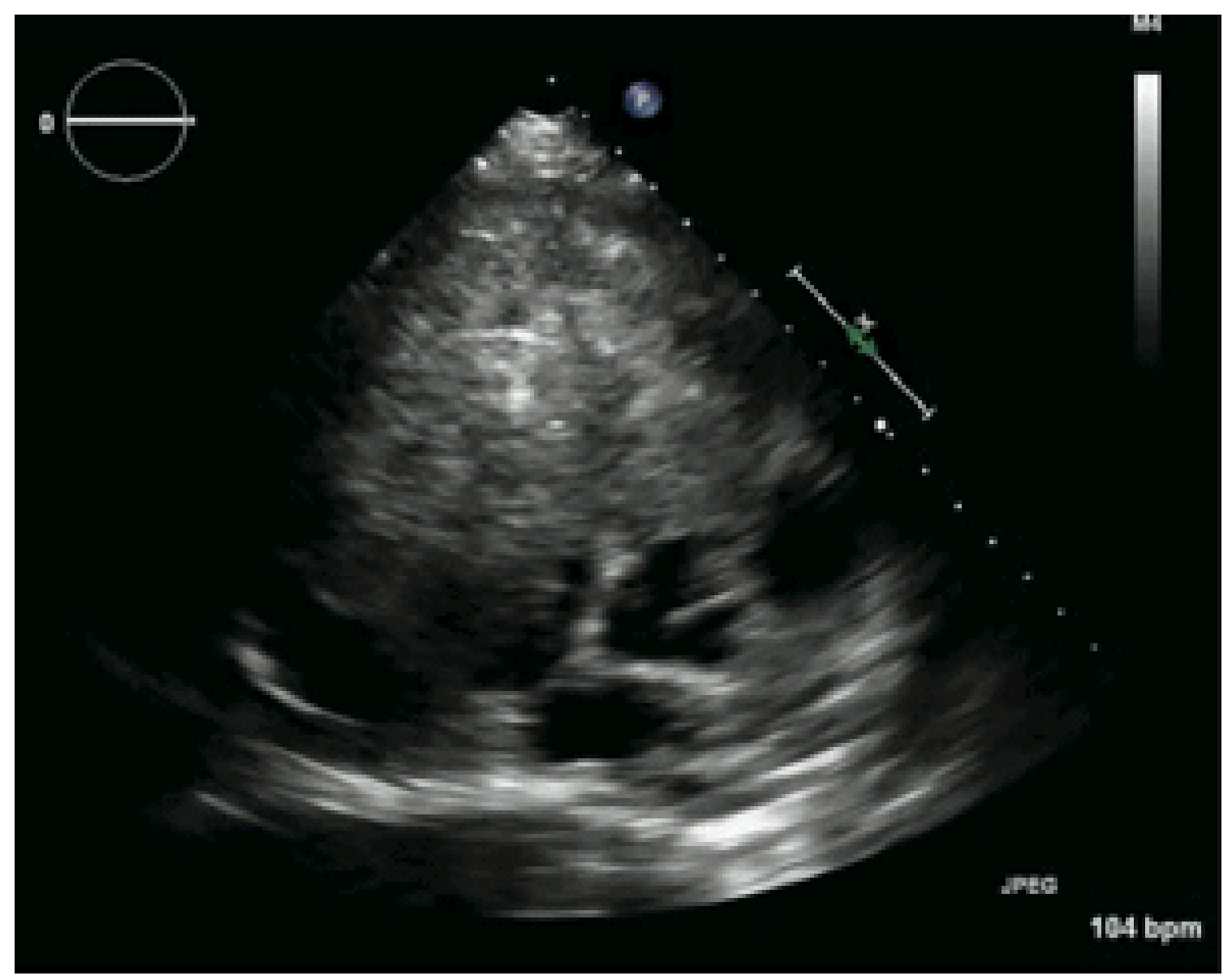

Figure 1. Parasternal short axis view at the level of the aortic valve 


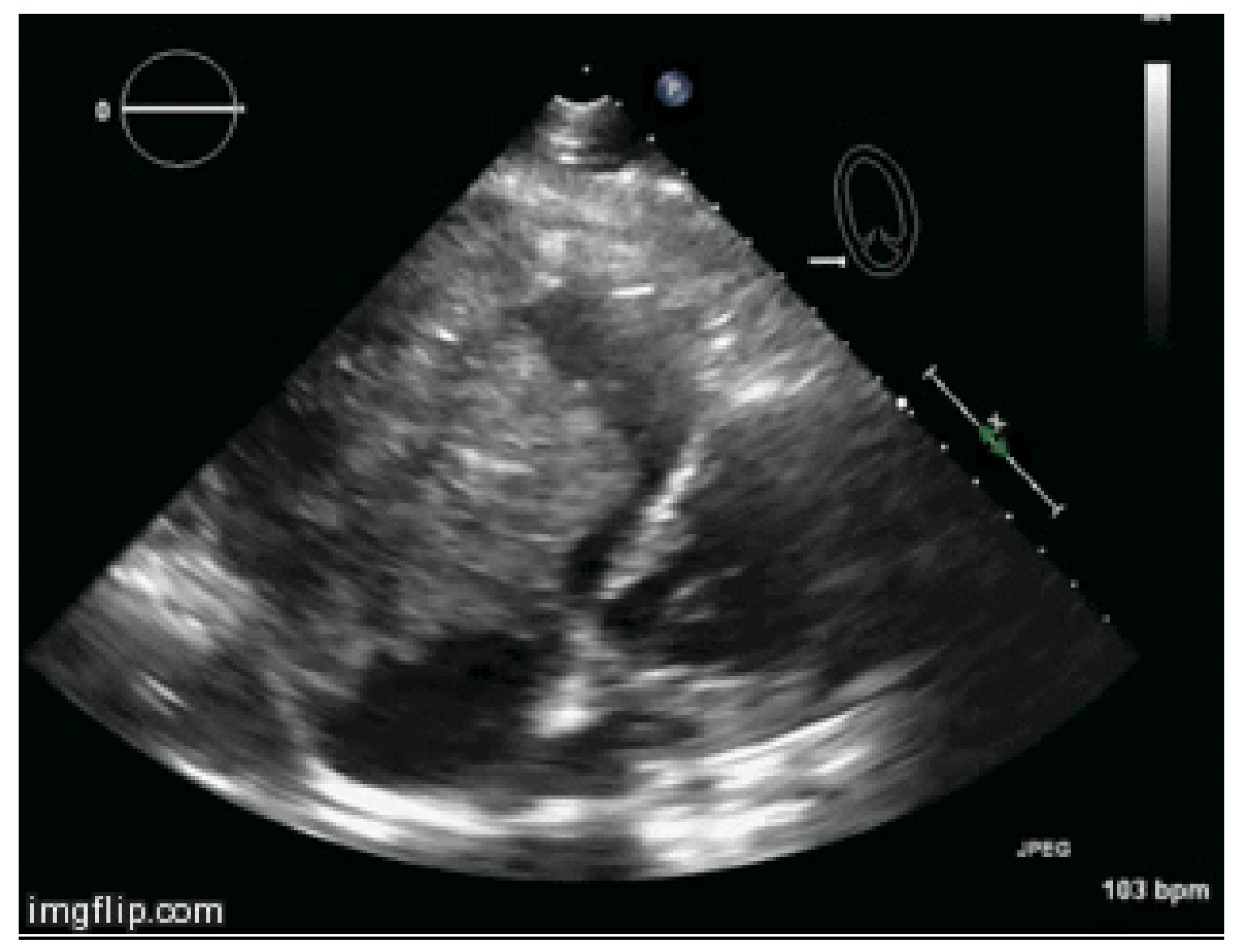

Figure 2. Apical 4 chamber view.

What is the best explanation for the echocardiographic findings shown above?

1. Atrial myxoma

2. Cardiac amyloidosis

3. Cardiac lymphoma

4. Takotsubo cardiomyopathy

5. Tricuspid valve endocarditis 


\section{Correct! \\ 3. Cardiac lymphoma}

The echocardiogram shows evidence of a mass nearly obliterating the entire right ventricle (almost completely obstructing flow thru the right ventricle) and partly occupying the right atrium. The left ventricle is compressed by the mass and hyperdynamic with an estimated ejection fraction of more than 70 percent (Figure 3).

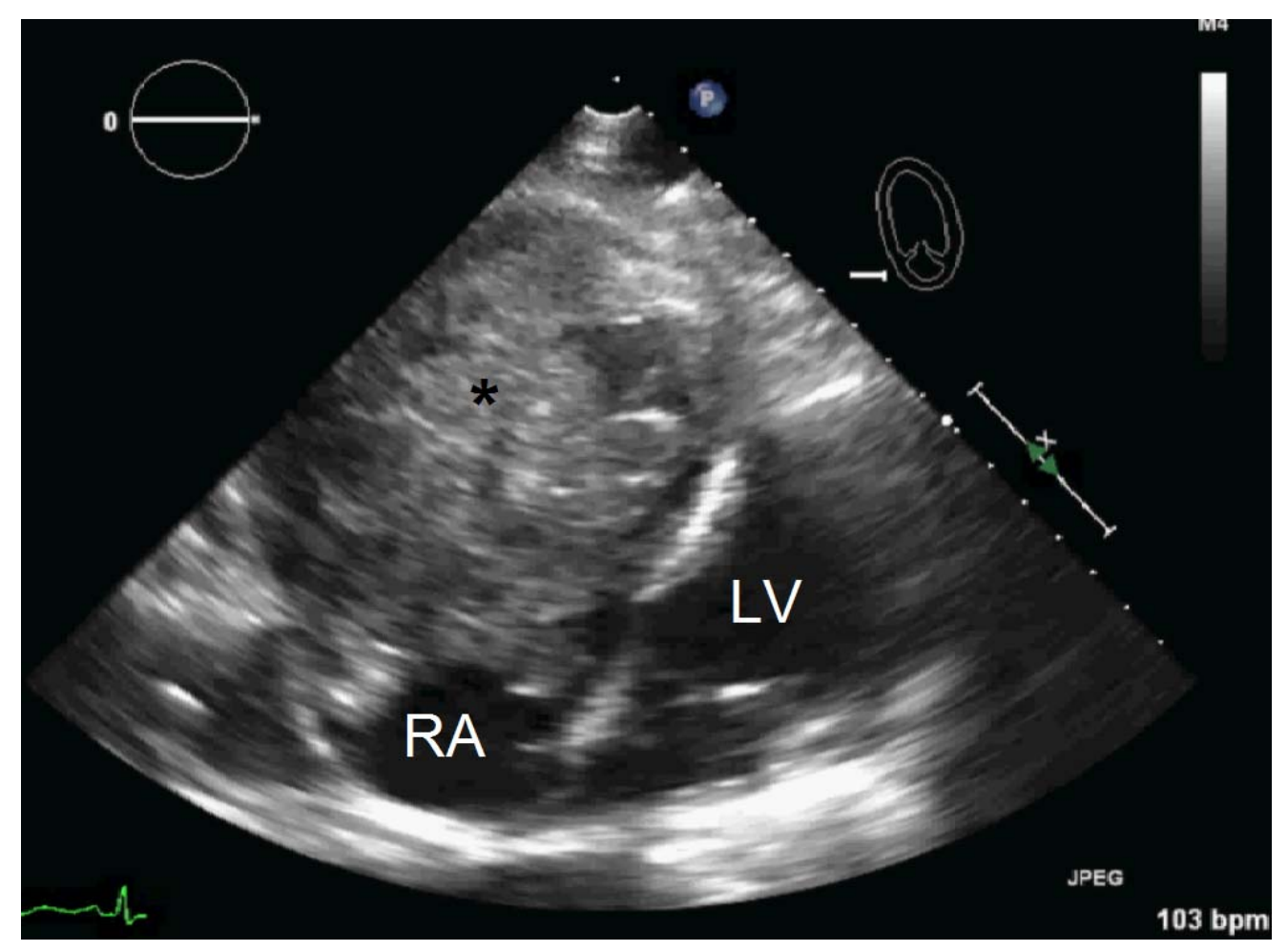

Figure 3. Apical 4 chamber echocardiogram image demonstrating mass $(*)$, and right atrium (RA) and left ventricle (LV) labeled.

It was felt the hypotension was due to restriction of flow around the mass, which was confirmed by Doppler studies. The patient was volume loaded to allow flow through the RV and placed on norepinephrine. Incidentally, bilateral retroperitoneal masses were found on abdominal ultrasound, which were biopsied and showed stage IV high grade diffuse large B-cell lymphoma.

Cardiothoracic surgery was consulted and determined that surgical resection was not recommended due to tumor extension into the right ventricular free wall. The patient underwent chemotherapy with R-CVD (rituximab, cyclophosphamide, vincristine and dexamethasone) for 2 weeks and a repeat echocardiogram showed reduction in the size of the cardiac mass (Figure 4). 


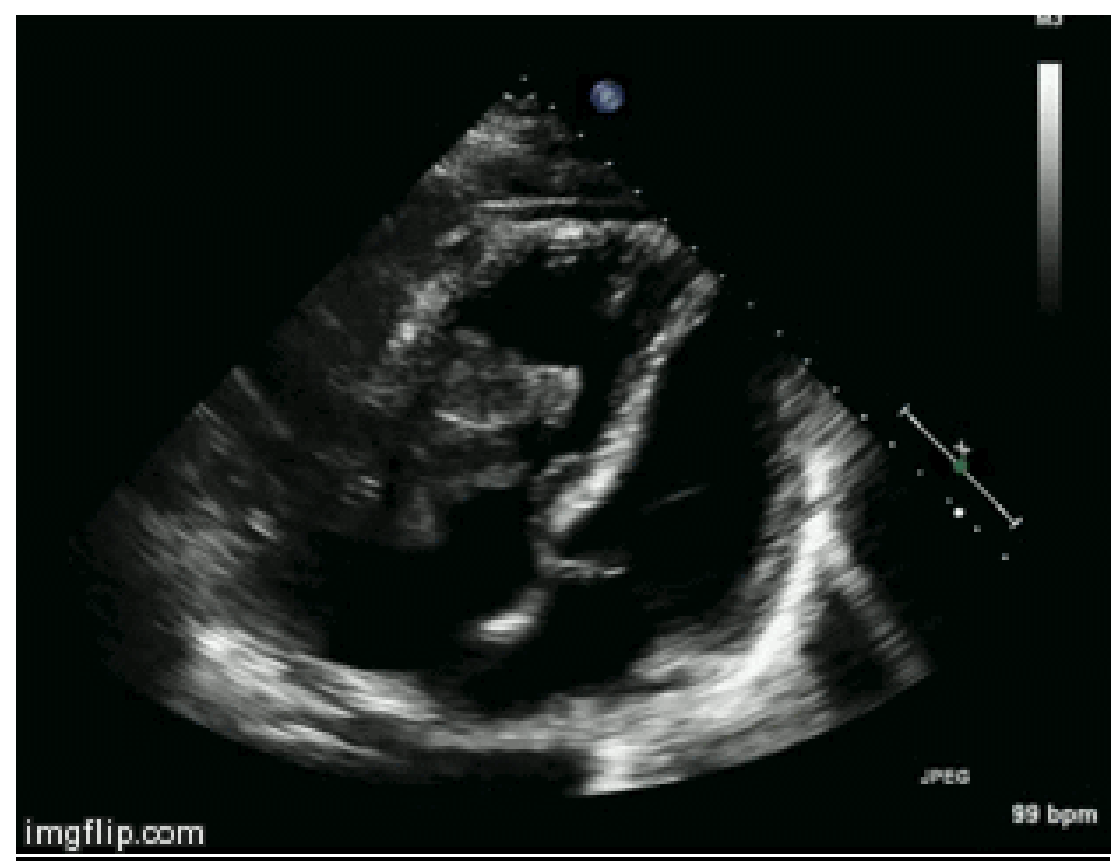

Figure 4. Apical 4 chamber view of the right ventricular mass, demonstrating reduction in the size of the mass after 1 cycle of chemotherapy.

The most common cardiac masses are atrial myxomas. Most typically, these are pedunculated round masses, attached to the interventricular septum, and located in the left atrium. They account for about $25 \%$ of all cardiac masses (1). Most cardiac masses are benign, with about a quarter being malignant (1). Of the malignant cardiac tumors only about $5 \%$ are primary cardiac lymphoma (as in this case). Primary cardiac lymphomas are typically of the diffuse large B-cell variant, and predominantly involve the right sided chambers, as demonstrated in this case $(1,2)$.

Incidental findings are frequently encountered on bedside echocardiography and have the potential to change clinical management and thought process. Literature recommends the routine use of bedside echocardiography by intensivists (3).

\section{References}

1. Klein Al, Asher CR. Cardiac Masses and Tumors in Clinical Echocardiography Review: A Self-Assessment Tool. $1^{\text {st }}$ ed. Philadelphia, PA: Lippincott, Williams \& Wilkins; 2011.

2. Antoniades L, Eftychiou C, Petrou PM, Bagatzounis A, Minas M. Primary cardiac lymphoma: case report and brief review of the literature. Echocardiography. 2009;26(2):214-9. [CrossRef] [PubMed]

3. Souza LF. Intensivists should use bedside echocardiography. Crit Care Med. 2005;33(12):2858-9. [CrossRef] [PubMed] 